\title{
Foot plantar pressure distribution modeling based on image processing
}

\begin{abstract}
Several factors have been associated with the distribution of plantar foot pressure, including: (i) the body weight, (ii) age, (iii) foot structure and (iv) standing / walking strategy. It is predicted that the biomechanics of the foot is influenced by the structure of the foot. The objective of this study was to obtain the plantar pressure distribution model of the foot using custom image processing algorithms upon the images captured by a commercial plantar pressure measurement machine, the EMED-X. The study involved the participation and data collection from 79 human subjects, ranging from age 201060 years old. This model can be analysed further to be used as a predictor for the formation of foot ulceration in certain subjects.
\end{abstract}

Keyword: Plantar pressure distribution; Modeling; Image processing; Foot ulceration 